\title{
Comparación de modelos alternativos en la evaluación genética de variables de crecimiento de ganado Brahman de registro en México ${ }^{\#}$
}

\author{
Comparison of alternative models for genetic evaluation of growth traits of \\ registered Brahman cattle in Mexico \\ GM Parra-Bracamonte ${ }^{\mathrm{a}^{*},}$ JC Martínez-González ${ }^{\mathrm{b}}$, EG Cienfuegos-Rivas ${ }^{\mathrm{b}}$, \\ A Tewolde-Medhin ${ }^{c}, \mathbf{R}$ Ramírez-Valverde ${ }^{\mathrm{d}}$ \\ aLaboratorio de Biotecnología Animal, Centro de Biotecnología Genómica, \\ Instituto Politécnico Nacional, Reynosa, Tamaulipas, México. \\ bDirección de Postgrado e Investigación, UAM Agronomía y Ciencias, \\ Universidad Autónoma de Tamaulipas, Victoria, Tamaulipas, México. \\ 'Dirección de Biotecnología y Bioseguridad - Instituto Interamericano \\ de Cooperación para la Agricultura, San José, Costa Rica. \\ dDepartamento de Zootecnia, Universidad Autónoma Chapingo, \\ Chapingo, Estado de México, México.
}

\begin{abstract}
SUMMARY
Five genetic evaluation models of registered Brahman cattle in Mexico were compared for birth weight (PN), weaning (PD), yearling (PA) and final weight at 550 days $(\mathrm{PF})$, and their effect on genetic parameter estimation and EPD ordering were also assessed. Models differed on number of random factors; D, model with direct genetic effect $\left(\sigma_{\mathrm{d}}^{2}\right)$, DM, model like D plus maternal genetic effect $\left(\sigma_{\mathrm{m}}^{2}\right)$ and genetic covariance between genetic effects equal to zero $\left(\sigma_{\mathrm{dm}}=0\right)$, DP, D like model plus maternal permanent effect $\left(\sigma_{\mathrm{p}}^{2}\right)$, DMC, DM like model plus covariance between genetic effects $\left(\sigma_{\mathrm{dm}} \neq 0\right)$, and DMCP as the complete model, equal to DMC plus $\left(\sigma_{\mathrm{p}}^{2}\right)$. Models included the fixed effect of contemporary grouping (herd, sex, year and season of birth), and age of dam as linear and quadratic covariable. A likelihood ratio test (PRV) was performed for model comparison, and EPD reordering was quantified by Spearman rank correlation analysis. The PRV showed DMC as the best model for all variables, except in PD. For PD the best model was DP. A negative genetic correlation $r_{d m}\left(r_{d m}=\sigma_{d m}{ }^{2}\right)$ was observed for PN, PA and PF $(-0,86,-0,84$ and $-0,52$, respectively) reducing the magnitude of total heritability estimates. For PD, DMC and DMCP did not converge. Correlation between selected models and the other compared models, suggested that there might be important and substantial changes on $10 \%$ of superior animals. Structure of data may modify the genetic parameter estimation if adjusting complex models; hence the model selection could be crucial before performing any genetic evaluation. Considerations were made concerning selective recording.
\end{abstract}

Palabras clave: Brahman, parámetros genéticos, modelos, DEPs. Key words: Brahman, genetic parameters, models, EPDs.

\section{INTRODUCCIÓN}

Para los productores de ganado bovino reproductor el objetivo primario es obtener un mejoramiento genético rápido y sostenido en su ganadería, para lo cual se utilizan herramientas de evaluaciones genéticas en las cuales la estimación de parámetros y valores genéticos confiables es el factor fundamental. Las evaluaciones confiables requieren de la aplicación de metodología adecuada y de las relaciones de parentesco entre animales (Van Vleck y col 1987). Para la predicción de valores genéticos utilizados

Aceptado: 25.03.2009.

\# Proyecto de investigación apoyado a través del convenio: SAGARPA2002-C01-0316 de los Fondos Sectoriales SAGARPA-CONAC y T.

* Centro de Biotecnología Genómica, Boulevard del Maestro S.N., Esq. Elías Piña, Col. Narciso Mendoza, Reynosa, Tamaulipas, México, C.P. 88710; gparra@ipn.mx rutinariamente en la selección, se requiere de la identificación de modelos estadísticos que maximicen la respuesta a la selección (Ramírez-Valverde y col 2007).

El modelo animal, mediante la metodología de la Mejor Predicción Lineal Insesgada (BLUP, por sus siglas en inglés), ha sido extensivamente utilizado para la evaluación genética en diferentes razas de ganado bovino para carne ${ }^{1}$ (Domínguez-Viveros y col 2003, Ferraz y col 2004), ajustando modelos de diferente complejidad para estimar valores genéticos que posteriormente son empleados para la selección de los reproductores.

Las variables de crecimiento son importantes en programas de selección de los sistemas de producción para carne (Goyache y Gutiérrez 2001, Ramírez-Valverde y col 2007),

\footnotetext{
Cabrera ME, A del V Garnero, RB Lôbo, RJ Gunski. 2001. Efecto de la incorporación de la covarianza genética directa-materna en el análisis de características de crecimiento en la raza Nelore. Livest Res Rural Develop (13)3 (http://www.cipav.org.co/lrrd/lrrd12/1/ cabr133.htm.)
} 
y la importancia relativa de los efectos genéticos aditivos, directos y maternos, debe ser considerada en la formulación de los planes de mejoramiento genético (Ferreira y col 1999), sobre todo para características medidas hasta el destete, las que son influidas en gran medida por los efectos maternos (Meyer 1992, Bijma 2006).

Varios estudios han sugerido que, aunque la capacidad de análisis no sea una limitante, el incluir o excluir algún efecto dentro del modelo de evaluación ocasiona la explicación incorrecta de los procesos o la incursión en un sesgo en la estimación y posterior en la jerarquización de los valores genéticos (Cabrera y col 2001, Domínguez-Viveros y col 2003, Ferraz y col 2004). Al respecto, Henderson (1975) mostró que al omitir del modelo algún efecto aleatorio importante o al incluir uno no importante, los estimadores de los efectos fijos y los predictores de los efectos aleatorios aún pueden considerarse insesgados; sin embargo, las varianzas de predicción del error de los efectos aleatorios se incrementan y eventualmente pueden provocar una menor respuesta a la selección.

El propósito de este estudio fue comparar cinco modelos de evaluación genética de variables de crecimiento en la población de ganado Brahman mexicano de registro, para determinar el modelo más apropiado en la estimación de parámetros genéticos y su impacto relativo en la predicción de valores genéticos.

\section{MATERIAL Y MÉTODOS}

\section{ORIGEN DE LA INFORMACIÓN}

La Asociación Mexicana de Criadores de Cebú (AMCC) estableció desde hace más de una década el programa de Control de Desarrollo Ponderal (CDP), bajo un esquema brasileño que consiste en el registro de variables de crecimiento de sementales de las diferentes razas Cebú que se registran, entre las cuales la raza Brahman es de las más populares y numerosas en México, y que actualmente cuenta con una cantidad significativa de registros produc- tivos que pueden proveer la información necesaria para conducir un programa de selección basado en parámetros y valores genéticos.

El presente estudio fue realizado con información de pesos de animales registrados en el CDP de la AMCC; para las variables de pesos al nacimiento $(\mathrm{PN})$, destete ajustado a $205 \mathrm{~d}$ (PD), año (PA) y peso final ajustado a 550 d (PF), de animales Brahman nacidos entre 1990 y 2003. El manejo en general de los animales ha sido descrito en publicaciones previas (AMCC 1996, Martínez 1999).

La base de datos original fue editada para ajustarla a los requerimientos del paquete de evaluaciones genéticas (MTDFREML, Boldman y col 1995). Para disminuir posibles errores de captura en la base de datos se eliminaron datos productivos extremos $(>3 \sigma)$ y los registros de animales que pudieron haber estado enfermos o que fueron tratados en forma preferencial. Las variables PD, PA y PF fueron ajustadas de acuerdo con lo recomendado por la Federación para el Mejoramiento del Ganado de Carne de los Estados Unidos (BIF 2002).

\section{ANÁLISIS ESTADÍSTICOS Y MODELOS ESTUDIADOS}

Los modelos para cada variable incluyeron el efecto fijo de grupo contemporáneo (hato, sexo del animal, año y época de nacimiento), del cual las épocas de nacimiento fueron definidas como: seca = enero-junio y de lluvias = julio-diciembre; y la covariable lineal y cuadrática de edad de la vaca.

Previo a los análisis genéticos, para cada característica se evaluaron las conexiones genéticas entre grupos contemporáneos utilizando el programa Milc.for (Fries 1998), cuyo fundamento es el uso de relaciones (conexiones) genéticas directas entre animales de diferentes grupos contemporáneos debidos a padres y madres comunes. Para considerar dos grupos contemporáneos conectados, el programa considera un mínimo de 10 conexiones genéticas directas. De esta evaluación se generaron las bases de datos de animales conectados genéticamente y que fueron utilizados en los análisis (cuadro 1).

Cuadro 1. Estructura y estadística descriptiva para las variables de crecimiento consideradas en los modelos de análisis.

Structure and descriptive statistics for analyzed growth traits.

\begin{tabular}{lrrrr}
\hline & \multicolumn{2}{c}{ Variables** } \\
\hline Concepto & PN & PD & PA & PF \\
\hline *Animales en A-1 & 38.559 & 31.553 & 29.458 & 26.881 \\
Sementales con cría & 1.469 & 1.331 & 1.291 & 1.268 \\
Vacas con cría & 15.148 & 12.921 & 12.332 & 11.507 \\
Crías por vaca & 1,6 & 1,6 & 1,5 & 1,5 \\
Media (kg) & 32,2 & 32,6 & 261,6 & 352,2 \\
Desviación Típica (kg) & 1,8 & 18,0 & 21,9 & 81,3 \\
Coefíciente de Variación (\%) & 5,7 & 23,1 \\
\hline
\end{tabular}

* $\quad \mathrm{A}^{1}=$ Matriz de relaciones de parentesco.

** PN, PD, PA y PF = Pesos al nacimiento, destete a los 205 d, año y 550 días. 
Los modelos en comparación consideraron el ignorar o incluir ciertos efectos aleatorios, quedando los cinco modelos siguientes:

1) $\mathrm{D}=$ Modelo que incluyó al efecto aditivo directo como único componente aleatorio.

2) $\mathrm{DP}=$ Modelo igual a $\mathrm{D}$, más el efecto materno de ambiente permanente.

3) $\mathrm{DM}=$ Modelo igual que $\mathrm{D}$, más el efecto aditivo materno y con $\sigma_{\mathrm{dm}}=0$.

4) $\mathrm{DMC}=$ Modelo igual a DM pero con $\sigma_{\mathrm{dm}} \neq 0, \mathrm{y}$

5) $\mathrm{DMCP}=$ Modelo igual que DMC, más el efecto materno de ambiente permanente (modelo completo).

En forma matricial el modelo completo puede describirse como:

$$
y=X \beta+Z d+W m+M p+e
$$

donde: $\mathbf{y}=$ es el vector de observaciones para PN, PD, PA o PF; $\mathbf{X}, \mathbf{Z}, \mathbf{W}$ y $\mathbf{M}=$ matrices conocidas de incidencia que relacionan las observaciones con sus respectivos vectores de efectos fijos y aleatorios; $\beta=$ es el vector de efectos fijos (grupo contemporáneo, y la covariable lineal y cuadrática de edad de la vaca); $\mathbf{d}=$ vector de efectos genéticos aditivos directos; $\mathbf{m}=$ vector de efectos genéticos aditivos maternos; $\mathbf{p}=$ el vector de efectos maternos de ambiente permanente y $\mathbf{e}=$ es el vector de efectos aleatorios residuales.

Los supuestos para el modelo, fueron:

$$
\mathrm{E}[y]=X b \mathrm{E}[d]=0, \mathrm{E}[m]=0, \mathrm{E}[p]=0, \mathrm{E}[e]=0,
$$

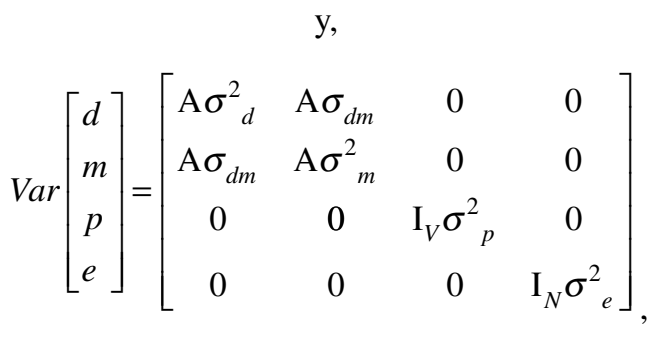

donde: $\mathbf{A}=$ matriz del numerador de relaciones de parentesco; $\mathbf{N}=$ número de observaciones; $\mathbf{V}=$ número de madres, e $\mathbf{I}=$ a las matrices identidad del orden correspondiente.

Los componentes de varianza y covarianza fueron estimados mediante el método de máxima verosimilitud restringida libre de derivadas (MTDFREML) (Boldman y col 1995). Los parámetros genéticos fueron estimados a partir de los componentes de (co)varianza obtenidos del mismo programa. El criterio de convergencia de los modelos fue considerado en $1 \times 10^{-13}$, y se realizaron tres reinicios en el análisis hasta que el cambio en el logaritmo de la función de verosimilitud fue menor a $1 \times 10^{-4}$, para asegurar la máxima global.

\section{COMPARACIÓN DE MODELOS}

Para la elección del modelo más apropiado, comparaciones estadísticas entre cada par de modelos (que difirieron en sólo un efecto aleatorio) fueron realizadas mediante la prueba de proporción de verosimilitudes (PRV), que se basa en las diferencias entre los logaritmos de las funciones de verosimilitud estimadas en el programa y comparadas con el valor de Chi-cuadrado $\left(\chi^{2}\right)$ con un grado de libertad al nivel de $5 \%$ de probabilidad.

La prueba, de acuerdo con Sorensen (2004) consideró:

$\mathrm{PRV}=(-2 \log \mathrm{L}$ modelo con $n$ parámetros $)-(-2 \log \mathrm{L}$ modelo con $n+1$ parámetros)

donde: $-2 \log \mathrm{L}=$ Logaritmo de verosimilitud.

Con las reglas de decisión:

Si PRV $\geq \chi_{(1, \alpha=0,05)}^{2}$, el modelo con $n+1$ parámetros se acepta significativamente como de mejor ajuste que el modelo con $n$ parámetros.

Si PRV $<\chi_{(1, \alpha=0,05)}^{2}$, los modelos con $n$ y $n+1$ parámetros no son significativamente diferentes.

Con el propósito de analizar el impacto del uso de los diferentes modelos en cada variable, los estimadores de los parámetros genéticos obtenidos con el modelo más apropiado fueron comparados con los otros modelos estudiados. En adición a lo anterior, el índice de herencia total $\left(\mathrm{h}_{\mathrm{T}}^{2}\right)$ fue calculado de acuerdo con lo propuesto por Willham (1972), como:

$$
\mathrm{h}_{\mathrm{T}}^{2}=\left(\sigma_{\mathrm{d}}^{2}+1,5 \sigma_{\mathrm{dm}}+0,5 \sigma_{\mathrm{m}}^{2}\right) / \sigma_{\mathrm{f}}^{2}
$$

donde: $\sigma_{\mathbf{d}}^{\mathbf{2}}=$ Varianza de efectos genéticos directos; $\sigma_{\mathbf{m}}^{2}=$ Varianza de efectos genéticos maternos; $\sigma_{\mathbf{d m}}=$ Covarianza entre efectos genéticos directos y maternos; $\mathrm{y} \sigma_{\mathbf{f}}^{2}=$ Varianza fenotípica.

\section{IMPACTO RELATIVO DEL USO DEL MODELO MÁS APROPIADO EN LA JERARQUIZACIÓN DE ANIMALES}

Para determinar el impacto de usar los modelos más apropiados respecto al uso de los otros modelos, se analizaron los cambios en la jerarquización de los mejores animales (10\% de los animales superiores) elegidos con el modelo más apropiado al ser evaluados con otros modelos. Coeficientes de correlación de rango (Spearman) fueron estimados considerando los valores genéticos de los animales superiores en los modelos más apropiados de cada variable y sus valores genéticos obtenidos con los otros modelos. Las correlaciones fueron estimadas entre valores genéticos directos y entre los maternos, usando el paquete estadístico SAS (SAS 2001).

\section{RESULTADOS}

En el cuadro 2 se presentan los valores de los logaritmos de las funciones de verosimilitud ( $-2 \log \mathrm{L})$ obtenidos en 
Cuadro 2. Componentes de (co)varianza* $\left(\mathrm{kg}^{2}\right)$ y logaritmos de verosimilitud (-2 Log L) para los modelos** utilizados en las variables de crecimiento de ganado Brahman de registro de México.

(Co)variance components and likelihood logarithms for models used in growth traits of registered Brahman cattle in Mexico.

\begin{tabular}{|c|c|c|c|c|c|c|c|}
\hline Modelo & $\sigma_{\mathrm{d}}^{2}$ & $\sigma_{m}^{2}$ & $\sigma_{\mathrm{dm}}$ & $\sigma_{p}^{2}$ & $\sigma_{\mathrm{e}}^{2}$ & $\sigma_{f}^{2}$ & $-2 \log \mathrm{L}$ \\
\hline \multicolumn{8}{|c|}{ Peso al nacimiento $(P N)$} \\
\hline $\mathrm{D}$ & 0,44 & & & & 1,66 & 2,10 & 41311,19 \\
\hline DP & 0,44 & & & 0,00 & 1,66 & 2,10 & 41311,19 \\
\hline $\mathrm{DM}$ & 0,44 & 0,00 & & & 1,66 & 2,10 & 41311,19 \\
\hline $\mathrm{DMC}^{\S}$ & 0,67 & 0,35 & $-0,42$ & & 1,53 & 2,14 & 41271,97 \\
\hline DMCP & 0,67 & 0,35 & $-0,41$ & 0,00 & 1,53 & 2,14 & 41271,97 \\
\hline \multicolumn{8}{|c|}{ Peso al destete ajustado a $205 d(P D)$} \\
\hline $\mathrm{D}$ & 217,18 & & & & 377,24 & 594,42 & 133065,34 \\
\hline DP & 133,45 & & & 44,34 & 402,12 & 579,91 & 133038,79 \\
\hline $\mathrm{DM}^{\S}$ & 129,74 & 44,82 & & & 404,83 & 579,38 & 133039,48 \\
\hline $\mathrm{DMC}^{\dagger}$ & - & - & - & & - & - & - \\
\hline $\mathrm{DMCP}^{\dagger}$ & - & - & - & - & - & - & - \\
\hline \multicolumn{8}{|c|}{ Peso al año (PA) } \\
\hline $\mathrm{D}$ & 347,17 & & & & 1035,74 & 1382,93 & 136441,51 \\
\hline DP & 297,51 & & & 36,40 & 1041,42 & 1375,33 & 136437,68 \\
\hline $\mathrm{DM}$ & 295,18 & 36,91 & & & 1042,93 & 1375,03 & 136437,37 \\
\hline $\mathrm{DMC}^{\S}$ & 339,02 & 269,36 & $-250,02$ & & 1022,14 & 1380,55 & 136423,77 \\
\hline $\mathrm{DMCP}$ & 338,79 & 256,04 & $-246,72$ & 10,52 & 1021,87 & 1380,50 & 136423,79 \\
\hline \multicolumn{8}{|c|}{ Peso final ajustado a $550 \mathrm{~d}(\mathrm{PF})$} \\
\hline $\mathrm{D}$ & 637,25 & & & & 1647,23 & 2284,48 & 130482,03 \\
\hline DP & 509,48 & & & 88,92 & 1666,24 & 2264,63 & 130476,55 \\
\hline DM & 489,26 & 101,92 & & & 1670,75 & 2261,93 & 130474,35 \\
\hline $\mathrm{DMC}^{\S}$ & 522,66 & 288,60 & $-202,12$ & & 1656,39 & 2265,53 & 130470,19 \\
\hline $\mathrm{DMCP}^{\dagger}$ & - & - & - & - & - & - & - \\
\hline
\end{tabular}

* $\sigma_{\mathrm{d}}^{2}=$ varianza del efecto genético aditivo directo, $\sigma_{\mathrm{m}}^{2}=$ varianza del efecto genético aditivo materno, $\sigma_{\mathrm{dm}}=$ covarianza genética entre efectos genéticos aditivos directos y maternos, $\sigma_{p}^{2}=$ varianza del efecto materno de ambiente permanente, $\sigma_{\mathrm{e}}^{2}=$ varianza residual, $\sigma_{\mathrm{f}}^{2}=$ varianza fenotípica.

** Componentes del modelo: $\mathrm{D}=$ Efecto directo, $\mathrm{M}=$ Efecto materno, $\mathrm{C}=$ Covarianza entre efectos directos y maternos, $\mathrm{P}=$ Efecto materno de ambiente permanente.

$\S \quad$ Mejor modelo de evaluación genética para la característica.

$\dagger$ Sin convergencia.

los modelos para cada una de las variables analizadas. Con la excepción de PD, la adición de los efectos maternos de ambiente permanente no mejoró el ajuste de los modelos $(\mathrm{P}>0,05)$, lo que se refleja en sus bajas proporciones en relación con la varianza fenotípica $(0,00$ a 0,04$)$. Para PN, PA y PF, la inclusión de la covarianza entre los efectos genético aditivo directo y materno (DMC) contribuyó $(\mathrm{P}<0,05)$ al mejor ajuste de los datos $(\mathrm{DM}, \mathrm{PRV}=39,2$; 13,6 y 4,1 ; respectivamente). Para las mismas variables no hubo mejoras importantes $(\mathrm{P}>0,05)$ o no se alcanzó la convergencia al adicionar el efecto materno de ambiente permanente (DMCP). Para PD, la inclusión del efecto genético aditivo materno (DM) o del materno de ambiente permanente (DP) explicaron mejor $(\mathrm{P}<0,05)$ la variabili- dad de los datos que al considerar sólo el efecto genético aditivo directo ( $\mathrm{D}, \mathrm{PRV}=25,9$ y 26,5 ; respectivamente), teniendo problemas de convergencia al adicionar otros efectos (DMC o DMCP).

Los valores en los estimadores de los componentes de (co)varianza y los parámetros genéticos fueron influidos por el modelo utilizado (cuadros 2 y 3 ). Los estimadores del índice de herencia directo $\left(\mathrm{h}^{2}{ }_{\mathrm{d}}\right)$ obtenidos de los modelos más apropiados oscilaron entre 0,23 y 0,32 y los del índice de herencia materno $\left(\mathrm{h}^{2}{ }_{\mathrm{m}}\right)$ entre 0,08 y 0,20 . Para PN, PA y PF, la adición del efecto genético aditivo materno o del materno de ambiente permanente al modelo D no modificaron sustancialmente los valores de los estimadores de los parámetros genéticos en su conjunto $\left(\mathrm{h}^{2}{ }_{\mathrm{T}}\right.$ 
Cuadro 3. Parámetros genéticos* para variables de crecimiento de ganado Brahman de registro de México.

Genetic parameters for growth traits of registered Brahman cattle in Mexico.

\begin{tabular}{|c|c|c|c|c|c|c|}
\hline Modelo & $\mathrm{h}_{\mathrm{d}}^{2}$ & $\mathrm{~h}_{\mathrm{m}}^{2}$ & $\mathrm{r}_{\mathrm{dm}}$ & $c^{2}$ & $\mathrm{~h}_{\mathrm{T}}^{2}$ & $\mathrm{e}^{2}$ \\
\hline \multicolumn{7}{|c|}{ Peso al nacimiento $(P N)$} \\
\hline $\mathrm{D}$ & 0,21 & & & & 0,21 & 0,79 \\
\hline DP & 0,21 & & & 0,00 & 0,21 & 0,79 \\
\hline DM & 0,21 & 0,00 & & & 0,21 & 0,79 \\
\hline $\mathrm{DMC}^{\S}$ & 0,32 & 0,16 & $-0,86$ & & 0,10 & 0,72 \\
\hline DMCP & 0,32 & 0,16 & $-0,86$ & 0,00 & 0,10 & 0,72 \\
\hline \multicolumn{7}{|c|}{ Peso al destete ajustado a $205 \mathrm{~d}(\mathrm{PD})$} \\
\hline D & 0,37 & & & & 0,37 & 0,63 \\
\hline DP & 0,23 & & & 0,07 & 0,23 & 0,69 \\
\hline $\mathrm{DM}^{\S}$ & 0,22 & 0,08 & & & 0,26 & 0,70 \\
\hline $\mathrm{DMC}^{\dagger}$ & - & - & - & & - & - \\
\hline $\mathrm{DMCP}^{\dagger}$ & - & - & - & - & - & - \\
\hline \multicolumn{7}{|c|}{ Peso al año (PA) } \\
\hline D & 0,25 & & & & 0,25 & 0,75 \\
\hline $\mathrm{DP}$ & 0,22 & & & 0,03 & 0,22 & 0,76 \\
\hline DM & 0,21 & 0,03 & & & 0,23 & 0,76 \\
\hline $\mathrm{DMC}^{\S}$ & 0,25 & 0,20 & $-0,83$ & & 0,07 & 0,74 \\
\hline $\mathrm{DMCP}$ & 0,25 & 0,19 & $-0,84$ & 0,01 & 0,07 & 0,74 \\
\hline \multicolumn{7}{|c|}{ Peso final ajustado a $550 \mathrm{~d}(\mathrm{PF})$} \\
\hline $\mathrm{D}$ & 0,28 & & & & 0,28 & 0,72 \\
\hline DP & 0,22 & & & 0,04 & 0,22 & 0,74 \\
\hline DM & 0,22 & 0,05 & & & 0,24 & 0,74 \\
\hline $\mathrm{DMC}^{\S}$ & 0,23 & 0,13 & $-0,52$ & & 0,16 & 0,73 \\
\hline $\mathrm{DMCP}^{\dagger}$ & - & - & - & - & - & - \\
\hline
\end{tabular}

* $\mathrm{h}^{2}{ }_{\mathrm{d}}=$ índice de herencia directo, $\mathrm{h}_{\mathrm{m}}^{2}=$ índice de herencia materno, $\mathrm{r}_{\mathrm{dm}}=$ correlación genética entre efectos genéticos aditivos directos y maternos, $\mathrm{c}^{2}=$ proporción de la varianza fenotípica debida a efectos maternos de ambiente permanente, $\mathrm{h}_{\mathrm{T}}^{2}=$ índice de herencia total $\left(h^{2}{ }_{T}=\sigma^{2}{ }_{d}+1.5 \sigma_{d m}+\right.$ $\left.0.5 \sigma_{m}^{2} / \sigma_{f}^{2}\right), \mathrm{e}^{2}=$ proporción de la varianza fenotípica debida a efectos ambientales residuales.

** Componentes del modelo: $\mathrm{D}=$ Efecto directo, $\mathrm{M}=$ Efecto materno, $\mathrm{C}=$ Covarianza entre efectos directos y maternos, $\mathrm{P} \approx=$ Efecto materno de ambiente permanente.

$\S \quad$ Mejor modelo de evaluación genética para la característica.

$\dagger$ Sin convergencia.

$=0,21 ; 0,22$ a 0,25 y 0,22 a 0,28 ; respectivamente). Los cambios mayores se observaron al incluir la covarianza entre efectos genéticos directo y materno $\left(\sigma_{\mathrm{dm}}\right)$, lo que se explica por los valores altos y negativos obtenidos de esos componentes de covarianza, y consecuentemente en las altas y negativas correlaciones entre estos efectos $\left(\mathrm{r}_{\mathrm{dm}}=-0,86\right.$; $-0,83$ y $-0,52$; respectivamente). Para PD, la inclusión del efecto genético aditivo materno o del materno de ambiente permanente provocó una disminución importante en los valores de los estimadores de $\operatorname{los} \mathrm{h}_{\mathrm{d}}^{2}(0,37$ vs $0,220,23$; para D vs DM o DP) y h ${ }_{\mathrm{T}}^{2}(0,37$ vs 0,26 y 0,23$)$.

En el cuadro 4 se muestran los coeficientes de correlación de rango estimados entre las diferencias esperadas en la progenie, predichos con el modelo más apropiado (de acuerdo con las PVR) y los predichos con los otros modelos estudiados. Las diferencias de los coeficientes de correlación con respecto al modelo de referencia oscilaron de 1 a 50\% para los valores genéticos directos y de 14 a $74 \%$ para los maternos.

\section{DISCUSIÓN}

\section{COMPARACIÓN DE MODELOS}

La baja proporción de crías por vaca (1,5 a 1,6; cuadro 1) en los datos, podría explicar la ausencia de mejora en el modelo al incluir el efecto materno de ambiente permanente en PN, PA y PF. Para PD, su inclusión mejoró el ajuste del modelo con sólo efectos genéticos aditivos directos (D vs DP); sin embargo, esto pudiera deberse a efectos confun- 
Cuadro 4. Coeficientes de correlación de rango (Spearman) para los valores genéticos estimados con el 10\% de los animales superiores elegidos con el modelo más apropiado y sus valores genéticos con los otros modelos.

Rank correlation coefficients (Spearman) among estimated genetic values of the top 10\% evaluated animals according to the most appropriate model and their estimated genetic values from the other models.

\begin{tabular}{lcccc}
\hline \multirow{2}{*}{ Modelos* } & \multicolumn{4}{c}{ Variables** (modelo más apropiado) } \\
\cline { 2 - 5 } & PN (DMC) & PD (DM) & PA (DMC) & PF (DMC) \\
\hline Entre valores genéticos directos & & & 0,50 & 0,68 \\
\hline D & 0,59 & 0,88 & 0,60 & 0,79 \\
DP & 0,59 & 0,99 & 0,60 & 0,81 \\
DM & 0,59 & - & - & - \\
DMC & - & 0,84 & 1,00 & 1,00 \\
DMCP & 1,00 & 0,92 & & 0,76 \\
\hline Entre valores genéticos maternos & & & - & - \\
\hline DM & 0,70 & - & 1,00 & 1,00 \\
DMC & - & 0,37 & 0,26 & \\
DMCP & 1,00 & & & \\
\hline
\end{tabular}

* Componentes de los modelos: $\mathrm{D}=$ Efecto directo, $\mathrm{M}=$ Efecto materno, $\mathrm{C}=$ Covarianza entre efectos directos y maternos, $\mathrm{P}=\mathrm{Efecto}$ materno de ambiente permanente.

** PN, PD, PA y PF = Pesos al nacimiento, destete a los 205 d, año y 550 días.

didos con los efectos genéticos aditivos maternos, ya que la adición de estos también mejoró en similar magnitud el uso de sólo efectos genéticos directos (D vs. DM), y su inclusión conjunta (DMCP) provocó la no convergencia del modelo. Problemas similares con la estructura de los datos han sido reportados por Domínguez-Viveros y col (2003), quienes encontraron en ganado Tropicarne que para la evaluación genética del PN, el mejor modelo fue el que omitió el efecto materno de ambiente permanente. En contraste, con mayor información en ganado Hereford, Angus y cruces de Cebú, en Australia, Meyer (1992) encontró que un modelo completo (DMCP) fue la mejor alternativa para la evaluación genética de variables de crecimiento.

Las PRV obtenidas en este estudio sugirieron como modelo más apropiado para PN, PA y PF al DMC; sin embargo, otros estudios de comparación de modelos para estas variables han producido diversidad en resultados. Para PN, en ganado Tropicarne, Domínguez-Viveros y col (2003) seleccionaron también a DMC; mientras que para Meyer (1992) el mejor modelo fue el DMCP. Para PA, Domínguez-Viveros y col (2003) eligieron como mejor modelo a DM, mientras que para Ferraz y col (2004) en ganado Tabapua, el mejor modelo fue DMCP. Para PF, Meyer (1992) encontró en ganado Angus que el modelo D ajustó mejor esta característica, mientras que Domínguez-Viveros y col (2003) observaron que el mejor modelo de ajuste fue DP. La diversidad en resultados es consecuencia de la diversidad en condiciones y estructura de los datos de cada estudio en particular, lo que refuerza la necesidad de realizar rutinariamente la determinación del modelo más apropiado en términos de los efectos aleatorios a incluir, como un paso previo a la publicación de resultados de la evaluación genética de poblaciones específicas.

En este estudio, la detección de efectos genéticos aditivos maternos importantes para PA y PF puede deberse a efectos residuales después del destete, lo que ha sido también reportado en otras investigaciones (Meyer 1992, Robinson 1996, Tosh y col 1999, Albuquerque y Meyer 2001, Ferraz y col 2004), y puede explicarse parcialmente en este caso por los bajos pesos de los animales (261,6 y 352,7 , para PA y PF, cuadro 1), los que pueden explicar aún influencia materna.

Los estimadores de los $\mathrm{h}_{\mathrm{d}}^{2} \mathrm{y} \mathrm{h}^{2}{ }_{\mathrm{m}}$ obtenidos con los modelos más apropiados fueron valores moderados para las variables estudiadas, y se encuentran dentro de los rangos publicados en literatura de bovinos para carne (Koots y col 1994). La estabilidad de los $\mathrm{h}_{\mathrm{d}}^{2} \mathrm{y} \mathrm{h}^{2}{ }_{\mathrm{T}}$ en PN, PD y PF al usar los modelos D, DM o DP, indica la escasa importancia práctica que tuvo la adición de sólo los efectos genéticos aditivos maternos o maternos de ambiente permanente; sin embargo, el incluir la $\sigma_{\mathrm{dm}}$ disminuyeron sustancialmente dichos parámetros, lo que sugiere posibles cambios de importancia práctica al obtener los valores genéticos con modelos con y sin incluir este efecto. Gutiérrez y col (2006) mencionaron que las estimaciones de efectos genéticos directos y maternos tienden a ser imprecisas debido a correlaciones de muestreo entre parámetros. La naturaleza de la variabilidad en las estimaciones de las correlaciones entre efectos directos y maternos no es clara. Algunos autores mencionan que en la estimación de dicho parámetro pueden existir sesgos por efecto del modelo utilizado y la estructura de los datos (Robinson 1996, Lee y Pollak 1997), mientras que otros consideran que se trata de un antagonismo real que genera 
estimaciones altas y negativas de este parámetro (Baker 1980, Meyer 1994).

Para PD en bovinos, varios estudios han mostrado la importancia del componente materno (Cundiff 1972, Gutiérrez y col 1997, Meyer 1997, Ferraz y col 2004, Bijma 2006). Considerando la posible confusión entre efectos genéticos aditivos maternos y maternos de ambiente permanente, y del reducido número de crías por vaca, en este estudio se seleccionó como modelo más apropiado a $\mathrm{DM}$. Al respecto, otros estudios (Gutiérrez y col 1997, Lôbo y col 2000, Domínguez-Viveros y col 2003) reportan la variedad en el grado de importancia de los componentes aleatorios cuando se evalúa esta variable. Similar a los resultados de este estudio, Domínguez-Viveros y col (2003) concluyeron que el mejor modelo para analizar PD fue DM, mientras que Ferraz y col (2004) seleccionaron a DMCP. Para esta variable, la dificultad de ajustar modelos incluyendo la covarianza entre efectos genéticos aditivos directos y maternos ha sido asociada a un efecto de la estructura de los datos (Gutiérrez y col 1997, Lôbo y col 2000) y deficiencias en los modelos de ajuste (Bijma 2006). En este estudio, la inclusión de la covarianza provocó la falta de convergencia del sistema de ecuaciones al utilizar los modelos DMC o DMCP, lo que puede atribuirse parcialmente a la estructura de los datos que conduce a la dificultad de obtener el valor del parámetro dentro de los límites estimables.

La mayoría de estudios en PD de bovinos para carne reportan estimaciones de $\mathrm{r}_{\mathrm{dm}}$ negativas, aunque también se han estimado con valores positivos o cercanos a cero (Meyer 1992, Eler y col 1995, Dodenhoff y col 1999, Tosh y col 1999, Albuquerque y Meyer 2001). Dada la falta de claridad en los mecanismos para estimar con precisión la covarianza entre efectos genéticos directo y materno del PD, 14 de 20 Asociaciones de Criadores de bovinos para carne de Estados Unidos utilizan un valor de cero en las evaluaciones genéticas nacionales (BIF 2002), es decir modelos DM.

Las explicaciones de la obtención de estimadores extremos de $\sigma_{\mathrm{dm}}$, se han centrado en la existencia de covarianzas ambientales entre registros de las vacas y sus progenies, y en la estructura de los efectos fijos usados en los modelos estadísticos para el análisis de los datos (Bijma 2006), lo anterior atribuido a causas como apareamientos diferenciales no-aleatorios entre hatos y diferencias en manejo no contabilizadas dentro de grupos contemporáneos, sugiriendo una posible confusión entre efectos genéticos y ambientales relacionada con los sementales, y provocando una sobreestimación de la varianza genética aditiva (Gutiérrez y col 2006). Las sugerencias para enfrentar esta particularidad incluyen contar con información productiva de tantas relaciones genéticas maternas como sea posible, tanto de las madres como de las abuelas maternas (Maniatis y Pollot 2003); adicionar un efecto aleatorio de interacción semental por año, por hato, por regiones o por grupo contemporáneo (Notter y col 1992, Robinson 1996, Lee y Pollak 1997, Dodenhoff y col 1999, Gutiérrez y col 2006); ajustar un modelo de semental-abuelo materno con el efecto de madre anidado en el efecto de abuelo materno (Gutiérrez y col 1997) o incluir la regresión sobre el fenotipo de la madre (Robinson 1996, Dodenhoff y col 1999, Bijma 2006).

Koots y col (1994) revisaron parámetros genéticos de poblaciones de bovinos para carne, mencionando que gran parte de la variabilidad en estimadores de los índices de herencia parece deberse a modelos estadísticos inapropiados más que a diferencias reales entre las poblaciones. El cuantificar lo más cercano a la realidad la importancia de la influencia materna y su correlación entre efectos directos y maternos, es esencial para la evaluación genética del PD. Según Ferraz y col (2004), si la correlación fuera positiva o cero, la selección basada en PD normalmente no sería un problema; sin embargo, si la correlación es negativa y alta provocaría disminución en el progreso genético, ya que parte de la ganancia obtenida por el aumento en el crecimiento del propio animal podría ser acompañada por una reducción en la habilidad materna de las vacas, y viceversa. Por lo anterior, se requiere de estudios más detallados que expliquen mejor la naturaleza del fenómeno y su tratamiento en condiciones específicas.

\section{IMPACTO RELATIVO DEL USO DEL MODELO MÁS} APROPIADO EN LA JERARQUIZACIÓN DE ANIMALES

La estimación de las correlaciones de rango para establecer el impacto relativo que podría tener el uso de un modelo diferente al apropiado bajo los supuestos del PRV, indicó que los valores genéticos pueden variar sensiblemente y hasta un 50\% para los valores genéticos directos y hasta un $74 \%$ para los valores genéticos maternos.

Si representamos el costo de una subestimación de valores genéticos en un esquema de mejoramiento genético basado en el uso del modelo animal como herramienta para la selección y compra de ejemplares en catálogos, un cambio en los valores genéticos estimados representaría pérdidas cuantiosas. Además, si el sobreprecio está basado en el mejor $10 \%$ de la población evaluada, el cambio en la jerarquización requerirá mayor atención a diferentes niveles de organización incluidas las ganaderías participantes, que comercializan el ganado reproductor diseminando de esta manera el progreso genético.

Se concluye que existe la necesidad de distinguir el modelo más apropiado para las evaluaciones genéticas de características de crecimiento, y sobre todo considerando que las condiciones particulares de la estructura de los datos analizados pueden representar un reto para el análisis e interpretación de los resultados al ajustar modelos complejos.

\section{RESUMEN}

Se realizó una comparación de modelos para la evaluación genética de variables de crecimiento, en los pesos al nacimiento (PN), destete (PD), año (PA) y 550 días (PF) de ganado Brahman de registro de México; 
con el propósito de cuantificar su efecto en la estimación de parámetros genéticos y la jerarquización de las diferencias esperadas de progenie estimadas (DEPs). Los modelos comparados diferían en componentes aleatorios y fueron, $\mathrm{D}$ con el efecto directo $\left(\sigma^{2}{ }_{\mathrm{d}}\right)$; DM, igual a D pero incluyendo el efecto materno $\left(\sigma_{\mathrm{m}}^{2}\right)$ y con la covarianza entre los efectos de $\sigma_{\mathrm{d}}^{2} \mathrm{y} \sigma_{\mathrm{m}}^{2}\left(\sigma_{\mathrm{dm}}\right)=0$; DP, como D más el efecto materno de ambiente permanente $\left(\sigma_{\mathrm{p}}^{2}\right)$; DMC, como DM pero con $\sigma_{\mathrm{dm}} \neq 0$; y el modelo completo, DMCP, igual a DMC más $\sigma_{\mathrm{p}}^{2}$. Además, incluyeron el efecto fijo de grupo contemporáneo (hato-sexo-año-época) y la covariable de edad de la madre (lineal y cuadrática). La comparación entre modelos se realizó mediante la prueba de proporción de verosimilitudes (PRV). Las DEPs de los modelos, se compararon por correlación (rango de Spearman). Según la PRV, en todas las variables, excepto PD, el mejor modelo de ajuste fue DMC. Para PD, el mejor modelo fue DP. En PN, PA y PF, se observó una $r_{d m}\left(r_{d m}=\right.$ $\left.\sigma_{\mathrm{dm}}\right)$ negativa $(-0,86,-0,84$ y $-0,52$, respectivamente); lo que disminuyó la magnitud de los estimadores de índice de herencia total. Para PD, los modelos DMC y DMCP no alcanzaron la convergencia. La estimación de las correlaciones de rango entre los valores genéticos directos y maternos de los modelos seleccionados con respecto a los otros modelos comparados, indicó que puede existir cambio sustantivo en la jerarquización del 10\% de la población con valores superiores. Se concluye que la estructura de los datos analizados puede afectar la estimación de parámetros genéticos en modelos complejos, por lo que es importante escoger el modelo apropiado antes de llevar al cabo una evaluación genética. Existen algunas consideraciones con respecto al registro selectivo que deben tomarse con reserva sobre todo en evaluaciones nacionales.

\section{AGRADECIMIENTOS}

Los autores desean expresar su agradecimiento a la Asociación Mexicana de Criadores de Cebú por facilitar el acceso a la base de datos analizada, y a los Fondos Sectoriales SAGARPA-CONACyT, por el apoyo financiero para llevar a cabo el proyecto de investigación a través del convenio SAGARPA-2002-C01-0316, del cual derivó este trabajo.

\section{REFERENCIAS}

Albuquerque GL, K Meyer. 2001. Estimates of direct and maternal genetic effects for weights from birth to 600 days of age in Nelore cattle. J Anim Breed Genet 118, 83-92.

AMCC. 1996. Reglamento Técnico del Control de Desarrollo Ponderal. Asociación Mexicana de Criadores de Cebú. Tampico, Tamaulipas.

Baker RL. 1980. The role of maternal effects on the efficiency of selection in beef cattle. A review. Proc N Z Soc Anim Prod 40, 285-303.

BIF. 2002. Uniform Guidelines for Beef Improvement Programs. Beef Improvement Federation. $8^{\text {th }}$ ed. Athens, Georgia, USA.

Bijma P. 2006. Estimating maternal genetic effects in livestock. J Anim Sci 84, 800-806.

Boldman KG, LA Kriese, LD Van Vleck, CP Van Tassell, SD Kachman. 1995. A Manual for Use of MTDFREML, a Set of Programs to Obtain Estimates of Variances and Covariances. United States Department of Agriculture, Agricultural Research Service, Clay Center, Lincoln, Nebraska, USA.

Cundiff LV. 1972. The role of maternal effects in animal breeding: VIII. Comparative aspects of maternal effects. J Anim Sci 35, 1335-1337.

Dodenhoff J, LD Van Vleck, DE Wilson. 1999. Comparison of models to estimate genetic effects for weaning weight of Angus cattle. J Anim Sci 77, 3176-3184.

Domínguez-Viveros J, R Núñez-Domínguez, R Ramírez-Valverde, A RuizFlores. 2003. Evaluación genética de variables de crecimiento en bovinos Tropicarne: Selección de modelos. Agrociencia 37, 323-335.

Eler JP, LD Van Vleck, JBS Ferraz, RB Lôbo. 1995. Estimation of variances due to direct and maternal effects for growth traits of Nelore cattle. J Anim Sci 73, 3253-3258.

Ferraz PB, A Ramos, LO da Silva, JC de Souza, MM de Alencar. 2004. Alternative animal models to estimate heritabilities and genetic correlations between direct and maternal effects of pre and post-weaning weights of Tapapuâ cattle. Arch Lat Prod Anim 12, 119-125.

Ferreira GB, MD MacNeil, LD Van Vleck. 1999. Variance components and breeding values for growth traits from different statistical models. J Anim Sci 77, 2641-2650.

Fries L. 1998. Connectability in Beef Cattle Evaluation: The heuristic approach used in MILC.FOR. In: Proceedings of the $6^{\text {th }}$ World Congress on Genetics Applied to Livestock Production. Armidale, Australia, Pp 449-450.

Goyache F, JP Gutiérrez. 2001. Heritability of reproductive traits in Asturiana de los Valles beef cattle breed. Arch Tierz Dummerstorf 44, 489-496.

Gutiérrez JP, J Cañón, F Goyache. 1997. Estimation of direct and maternal genetic parameters for preweaning traits in the Asturiana de los Valles beef cattle through animal and sire models. J Anim Breed Genet 114, 261-266.

Gutiérrez JP, I Fernández, I Alvarez, LJ Royo, F Goyache. 2006. Sire x contemporary group interactions for birth weight and preweaning growth traits in the Asturiana de los Valles beef cattle breed. Livest Sci 99, 61-68.

Henderson CR. 1975. Comparison of alternative sire evaluation methods. J Anim Sci 41, 760-770.

Koots KR, JP Gibson, JW Wilton. 1994. Analysis of published genetic parameter estimates for beef production traits. 2. Phenotypic and genetic correlations. Anim Breed Abst 62, 825-853.

Lee C, EJ Pollak. 1997. Relationship between sire x year interactions and direct-maternal genetic correlation for weaning weight of simmental cattle. J Anim Sci 75, 68-75.

Lôbo RNB, RF Martins, VM Penna, MF Lima de A. 2000. Genetic parameters for growth traits of zebu cattle in the semi-arid region of Brazil. Ciência Anim 10, 7-12.

Maniatis N, GE Pollot. 2003. The impact of data structure on genetic (co)variance components of early growth in sheep, estimated using an animal model with maternal effects. J Anim Sci 81, 101-108.

Martínez GJC. 1999. Tendencias fenotípicas, genéticas y ambientales de características de crecimiento en el ganado Cebú. Tesis Doctoral. Universidad Autónoma de Tamaulipas, Cd. Victoria, Tamaulipas, México.

Meyer K. 1992. Variance components due to direct and maternal effects for growth traits of Australian beef cattle. Livest Prod Sci 31, 179-204.

Meyer K. 1994. Estimates of direct and maternal correlations among growth traits in Australian beef cattle. Livest Prod Sci 38, 91-105.

Meyer K. 1997. Estimates of genetic parameters for weaning weight of beef cattle accounting for direct-maternal environmental covariances. Livest Prod Sci 52, 187-199.

Notter DR, B Tier, K Meyer. 1992. Sire x herd interactions for weaning weight in beef cattle. J Anim Sci 70, 2359-2365.

Ramírez-Valverde R, OC Hernández-Alvarez, R Núñez-Domínguez, A Ruíz-Flores, JG García-Muñiz. 2007. Análisis univariado vs multivariado en la evaluación genética de variables de crecimiento en dos razas bovinas. Agrociencia 41, 271-282.

Robinson DL. 1996. Models which might explain negative correlations between direct and maternal genetic effects. Livest Prod Sci 45, 111-122.

SAS. 2001. SAS/STAT User's Guide (Release 8.20). SAS Inst. Inc. Cary, NC, USA.

Sorensen D. 2004. An Introductory Overview of Model Comparison and Related Topics. On short course: Model choice, model assessment, and related topics, from a likelihood to Bayesian perspective. Department of Genetics. Danish Institute of Agricultural Sciences, Tjele, Denmark.

Tosh JJ, RA Kemp, DR Ward. 1999. Estimates of direct and maternal genetic parameters for weight traits and backfat thickness in a multibreed population of beef cattle. Can J Anim Sci 79, 433-439.

Van Vleck LD, EJ Pollack, EA Branford-Oltenacu. 1987. Genetics for the Animal Sciences. W.H. Freeman and Company, USA.

Willham RL. 1972. The role of maternal effects in animal breeding: III. Biometrical aspects of maternal effects in animals. J Anim Sci 35, 1288-1293. 